\title{
Dynamics of quantum dissipation systems interacting with bosonic canonical bath: Hierarchical equations of motion approach
}

\author{
Rui-Xue $\mathrm{Xu}$ and YiJing Yan \\ Hefei National Laboratory for Physical Sciences at the Microscale, University of Science and Technology of China, Hefei, China \\ and Department of Chemistry, Hong Kong University of Science and Technology, Kowloon, Hong Kong, China
}

(Received 24 October 2006; revised manuscript received 11 December 2006; published 15 March 2007)

\begin{abstract}
A nonperturbative theory is developed, aiming at an exact and efficient evaluation of a general quantum system interacting with arbitrary bath environment at any temperature and in the presence of arbitrary timedependent external fields. An exact hierarchical equations of motion formalism is constructed on the basis of a calculus-on-path-integral algorithm, via the auxiliary influence generating functionals related to the interaction bath correlation functions in a parametrization expansion form. The corresponding continued-fraction Green's functions formalism for quantum dissipation is also presented. Proposed further is the principle of residue correction, not just for truncating the infinite hierarchy, but also for incorporating the small residue dissipation that may arise from the practical difference between the true and parametrized bath correlation functions. The final residue-corrected hierarchical equations of motion can therefore be used practically for the evaluation of arbitrary dissipative quantum systems.
\end{abstract}

DOI: 10.1103/PhysRevE.75.031107

PACS number(s): 05.30.- d, 05.40.-a

\section{INTRODUCTION}

As a fundamental topic in quantum statistical mechanics, the development of quantum dissipation theory has involved scientists from diversified fields of research over decades [1-30]. The key quantity in quantum dissipation theory is the reduced density operator $\rho(t) \equiv \operatorname{tr}_{\mathrm{B}} \rho_{\mathrm{T}}(t)$; i.e., the partial trace of the total density operator over the bath space of practically infinite degrees of freedom. The standard approach to the exact $\rho(t)$ is the influence functional path integral formalism [1]. However, from both operational and numerical points of view, this formally exact formalism is much limited in comparison with its differential or master equation counterpart.

An attempt at the construction of a quantum master equation, via the derivative on the exact path integral formalism, was first carried out by Caldeira and Leggett, for quantum dissipation in the high-temperature Markovian limit [11]. The calculus-on-path-integral (COPI) method has also been used in driven bistable systems, together with the so-called noninteracting blip approximation or its variations to treat the quantum path correlations in the reduced dynamics $[4,8,12]$. The first exact quantum master equation via the COPI method was constructed for damped harmonic oscillator systems [31,32]. Ishizaki and Tanimura have recently constructed a set of hierarchically coupled quantum FokkerPlanck equations for a general Drude dissipation system [21], which no longer exploits the high-temperature approximation as before $[19,20]$. The deterministic description of an exact differential equation of motion (EOM) formalism can also be constructed via stochastic descriptions of quantum dissipation [22-29]. However, it has thus far only been carried out with a certain single-mode dissipation model system. The general theory of quantum dissipation in terms of a deterministic EOM remains a great challenge.

The aim of this work is to deal with this fundamental issue of quantum statistical mechanics. It is to develop an exact and efficient EOM formalism for a general quantum system, interacting with arbitrary bath environment at any temperature and arbitrary time-dependent external fields. The only assumption involved is the same as in the path integral formalism: that the interaction bath satisfy Gaussian statistics $[1,4,17]$. The theoretical construction will be made mainly based on the COPI algorithm developed in Ref. [17]. A set of so-called influence generating functionals will be shown to be crucial in formulating the exact hierarchical EOM. The present work focuses on the canonical bath ensemble case, but can be easily extended to the grand canonical case [33].

The remainder of this paper is organized as follows. In Sec. II, after the description of the general form of the system-bath coupling Hamiltonian, we review the path integral influence functional formalism [17], together with its formal relation to the time-local dissipation superoperator in the quantum master equation. In Sec. III, we illustrate with the Drude-Debye model the key technical issue of the construction of a hierarchical EOM. It is to identify a complete set of auxiliary influence generating functionals (IGF's) via the COPI algorithm [17]. Section IV turns to general dissipative quantum systems. It involves a parametrization of general interaction bath correlation functions that satisfy the fluctuation-dissipation theorem; see Appendix A for details. Upon identifying the complete set of auxiliary IGF-COPI constructions for the parametrized forms of correlation functions, exact hierarchical EOM follow immediately. The equivalent formalism in terms of the hierarchical and continued-fraction Green's functions and memory kernels is given in Appendix B. In Sec. V, we further establish the so-called principle of residue correction. The principle itself is rooted at the formally exact relation established in Sec. II between the Feynman-Vernon influence functional and the time-local dissipation superoperator. But it is now exploited in a certain perturbative or nonperturbative approximate manner to construct an efficient hierarchy truncation method. It is also used to incorporate the residue contribution, due to the difference between the exact and parametrized baths, into the final theory. Finally, Sec. VI concludes this paper. 


\section{QUANTUM DISSIPATION IN TERMS OF INFLUENCE FUNCTIONALS}

\section{A. Multimode system-bath coupling Hamiltonian}

Consider a general quantum system embedded in a bath which is assumed as a canonical ensemble in this work. The total system-plus-bath composite Hamiltonian can be written in general as

$$
H_{\mathrm{T}}=H(t)-\sum_{a} Q_{a} \hat{F}_{a}+h_{\mathrm{B}}
$$

Here, $H(t)$ and $h_{B}$ denote the uncorrelated system and bath Hamiltonians, respectively. The former may be subject to a time-dependent coherent field drive. The second term on the right-hand side (RHS) of Eq. (1) denotes the multimode system-bath coupling. It can be generally expressed in the multiple-dissipative-mode decomposition form, in which $\left\{Q_{a}\right\}$ and $\left\{\hat{F}_{a}\right\}$ are the system and bath operators, respectively, and assumed to be Hermite in this work.

For later use, we introduce here the Liouvillian $\mathcal{L}$ and the dissipative coupling mode $\mathcal{Q}_{a}$ in the reduced system subspace via their actions on an arbitrary operator as

$$
\mathcal{L} \hat{O} \equiv[H(t), \hat{O}], \quad \mathcal{Q}_{a} \hat{O} \equiv\left[Q_{a}, \hat{O}\right]
$$

Throughout this work, we set $\hbar \equiv 1$ and the inverse temperature $\beta \equiv 1 /\left(k_{B} T\right)$, and denote also $\partial_{t} \equiv \partial / \partial t$.

The stochastic canonical bath ensemble average of an operator $\hat{O}$ is denoted as

$$
\langle\hat{O}\rangle_{\mathrm{B}} \equiv \operatorname{tr}_{\mathrm{B}}\left(\hat{O} \rho_{\mathrm{B}}^{\mathrm{eq}}\right)=\operatorname{tr}_{\mathrm{B}}\left(\hat{O} e^{-\beta h_{\mathrm{B}}}\right) / \operatorname{tr}_{\mathrm{B}} e^{-\beta h_{\mathrm{B}}} .
$$

In the $h_{\mathrm{B}}$-interaction picture,

$$
\hat{F}_{a}(t) \equiv e^{i h_{\mathrm{B}} t} \hat{F}_{a} e^{-i h_{\mathrm{B}} t},
$$

for each bath interaction operator in Eq. (1), is assumed to be a Gaussian stochastic process. This is exactly the case when the bath consists of a collection of harmonic oscillators and each $\hat{F}_{a}$ is a linear combination of the coordinates and momenta of the harmonic bath oscillators. The Gaussian stochastic process is also related to the central limit theorem in statistics. As the quantity $Q_{a}\left\langle\hat{F}_{a}\right\rangle_{\mathrm{B}}$, if it is not zero, can be included in the system Hamiltonian in Eq. (1), we can set $\left\langle\hat{F}_{a}\right\rangle_{\mathrm{B}}=0$ without loss of generality. The effects of Gaussian stochastic bath operators are then completely described by their correlation functions,

$$
C_{a b}(t-\tau)=\left\langle\hat{F}_{a}(t) \hat{F}_{b}(\tau)\right\rangle_{\mathrm{B}} .
$$

They satisfy the symmetry and detailed-balance relations of the canonical bath $[4,16]$,

$$
C_{a b}^{*}(t)=C_{b a}(-t)=C_{a b}(t-i \beta) .
$$

We shall be interested in a differential EOM formalism, aiming at an efficient and exact evaluation of the reduced dynamics, with arbitrary multimode non-Markovian dissipation and time-dependent external fields on the system. The key quantity of interest is the reduced density operator $\rho(t)$, defined together with its associating propagator $\mathcal{U}\left(t, t_{0}\right)$ as

$$
\rho(t) \equiv \operatorname{tr}_{\mathrm{B}} \rho_{\mathrm{T}}(t) \equiv \mathcal{U}\left(t, t_{0}\right) \rho\left(t_{0}\right)
$$

The desired EOM will be constructed (cf. Sec. IV) via the IGF-COPI approach [17], starting from the exact path integral expression that involves only the initial factorization ansatz $\rho_{\mathrm{T}}\left(t_{0}\right)=\rho\left(t_{0}\right) \rho_{\mathrm{B}}^{\mathrm{eq}}$. Note that when the initial time is set to $t_{0} \rightarrow-\infty$, this ansatz becomes exact $[4,16]$.

\section{B. Reduced dynamics versus influence functionals}

This subsection summarizes the path integral formalism of quantum dissipation for the multimode system-bath interaction [17]. Exploited explicitly is only the Gaussian statistical property, the essence of the linear harmonic bath coupling with arbitrary system operators $\left\{Q_{a}\right\}$. Unlike the EOM formalism, which can be expressed at the operator level, the path integral expression goes with a representation. Let $\{|\alpha\rangle\}$ be a basis set in the system subspace. In the $\alpha$ representation, Eq. (7), reads [setting $\boldsymbol{\alpha} \equiv\left(\alpha, \alpha^{\prime}\right)$ for abbreviation]

$$
\rho(\boldsymbol{\alpha}, t) \equiv \rho\left(\alpha, \alpha^{\prime}, t\right)=\int d \boldsymbol{\alpha}_{0} \mathcal{U}\left(\boldsymbol{\alpha}, t ; \boldsymbol{\alpha}_{0}, t_{0}\right) \rho\left(\boldsymbol{\alpha}_{0}, t_{0}\right) .
$$

The reduced Liouville-space propagator reads in terms of the path integral as [1]

$$
\mathcal{U}\left(\boldsymbol{\alpha}, t ; \boldsymbol{\alpha}_{0}, t_{0}\right)=\int_{\boldsymbol{\alpha}_{0}\left[t_{0}\right]}^{\boldsymbol{\alpha}[t]} \mathcal{D} \boldsymbol{\alpha} e^{i S[\alpha]} \mathcal{F}[\boldsymbol{\alpha}] e^{-i S\left[\alpha^{\prime}\right]} .
$$

The effect of system-bath interaction on the reduced system dynamics is described by the Feynman-Vernon influence functional $\mathcal{F}$, which will be elaborated on soon. In Eq. (9), $S[\alpha]$ is the classical action functional of the reduced system, evaluated along the path $\alpha(\tau)$, with the constraints of the two ending points $\alpha\left(t_{0}\right)=\alpha_{0}$ and $\alpha(t)=\alpha$ being fixed. In the absence of bath interaction $(\mathcal{F}=1)$, the dynamics would be completely coherent; i.e., $\partial_{t} \mathcal{U}=-i \mathcal{L U}$ if $\mathcal{F}=1$.

Consider now the key quantity, the bath interactioninduced influence functional $\mathcal{F}$. Traditionally, its expression is derived by adopting a single-mode system-bath interaction model $[1,4]$, in which the bath $h_{B}$ is assumed to consist of a set of uncoupled harmonic oscillators $\left\{q_{j}\right\}$ and the systembath interaction assumes the form of $H^{\prime}=-Q \hat{F}=-Q \Sigma_{j} c_{j} q_{j}$, rather than the multimode decomposition as the second term of Eq. (1).

In connection with the later development of EOM formalism, we denote $\boldsymbol{a}=\left(a a^{\prime}\right)$ for a pair of dissipation modes hereafter and introduce [cf. the Eqs. (5) and (6) of Ref. [17]]

$$
\widetilde{\mathcal{Q}}_{\boldsymbol{a}}(t ;\{\boldsymbol{\alpha}\}) \equiv \widetilde{Q}_{a a^{\prime}}(t ;\{\alpha\})-\widetilde{Q}_{a a^{\prime}}^{\prime}\left(t ;\left\{\alpha^{\prime}\right\}\right),
$$

where (noting that $\widetilde{Q}_{a} \equiv \widetilde{Q}_{a a^{\prime}}$ and $C_{a} \equiv C_{a a^{\prime}}$ )

$$
\begin{gathered}
\tilde{Q}_{a}(t ;\{\alpha\}) \equiv \int_{t_{0}}^{t} d \tau C_{a}(t-\tau) Q_{a^{\prime}}[\alpha(\tau)], \\
\tilde{Q}_{a}^{\prime}\left(t ;\left\{\alpha^{\prime}\right\}\right) \equiv \int_{t_{0}}^{t} d \tau C_{a}^{*}(t-\tau) Q_{a^{\prime}}\left[\alpha^{\prime}(\tau)\right] .
\end{gathered}
$$




$$
\mathcal{Q}_{a}[\boldsymbol{\alpha}(t)] \equiv Q_{a}[\alpha(t)]-Q_{a}\left[\alpha^{\prime}(t)\right] .
$$

It is in fact the $Q_{a}$ commutator [cf. Eq. (2), the second identity] in the path integral representation, as it depends only on the fixed ending points. The final expression of the influence functional reads [17]

$$
\mathcal{F}[\boldsymbol{\alpha}] \equiv \exp \left\{-\int_{t_{0}}^{t} d \tau \mathcal{R}[\tau ;\{\boldsymbol{\alpha}\}]\right\},
$$

with

$$
\mathcal{R}[t ;\{\boldsymbol{\alpha}\}] \equiv \sum_{\boldsymbol{a}} \mathcal{Q}_{a}[\boldsymbol{\alpha}(t)] \widetilde{\mathcal{Q}}_{a}(t ;\{\boldsymbol{\alpha}\}) .
$$

The above relations will be used together with Eqs. (10)-(12) in the following sections to develop the desired EOM.

\section{Influence functional versus dissipation superoperator}

Note that in Eq. (13) the conventional influence phase functional is now expressed in terms of its time integrand $\mathcal{R}$. The latter is in fact the time-local dissipation superoperator $\mathcal{R}(t)$ in the path integral representation, as the time derivative of Eq. (9) with Eq. (13) leads to

$$
\partial_{t} \mathcal{U}=-i \mathcal{L U}-\mathcal{R}(t) \mathcal{U}
$$

or, equivalently,

$$
\dot{\rho}=-i \mathcal{L} \rho-\mathcal{R}(t) \rho .
$$

We can therefore call $\mathcal{R}$ of Eq. (13b) the dissipation functional. As inferred from Eq. (13b) with Eqs. (10) and (12), it may be symbolically expressed in the operator level as

$$
\mathcal{R} \hat{O}=\sum_{a}\left[Q_{a}, \widetilde{Q}_{a} \hat{O}-\hat{O} \widetilde{Q}_{a}^{\dagger}\right]
$$

Here, $\widetilde{Q}_{a}$ denotes the operator form of Eq. (11a). However, the explicit operator-level expression for $\mathcal{R}$ (or $\widetilde{Q}_{a}$ ) is generally not available. In the presence of a time-dependent external field, the only case being of the analytical expression of $\mathcal{R}$ (or $\widetilde{Q}_{a}$ ), is, to our best knowledge, the driven Brownian oscillator system [16].

The formal relations, Eqs. (13)-(15), also highlight where the difficulty is in the exact evaluation of quantum dissipation; all relate to the memory-containing $\widetilde{Q}$ or equivalent $\widetilde{\mathcal{Q}}$ functionals. In a certain sense, the EOM formalism to be presented in detail soon (cf. Secs. III and IV) is a mathematical construction that hierarchically resolves the "history" containing in the $\widetilde{\mathcal{Q}}$ functionals. This issue will become evident in the coming sections. Moreover, these formal relations may also be directly useful for the residue correction of the final formalism due to either hierarchy truncation or the small difference between the exact but complicated bath correlation functions and the parametrized ones (cf. Sec. V).

\section{HIERARCHICAL EQUATIONS OF MOTION: CORRELATED DEBYE DISSIPATION}

Before presenting the formalism for arbitrary dissipative systems, let us consider in this section the simplest multi- mode dissipation case, the Drude-Debye model, in which

$$
C_{a}(t>0)=C_{a a^{\prime}}(t)=\eta_{a} e^{-\gamma_{a} t} .
$$

The parameters $\gamma_{a} \equiv \gamma_{a a^{\prime}}=\gamma_{a^{\prime} a}$ are real, while $\eta_{a} \equiv \eta_{a a^{\prime}}$ $=\eta_{a^{\prime} a}^{*}$ are complex, and they are the same as the Drude parameters $\gamma_{\mathrm{D}}^{a}$ and $\eta_{\mathrm{D}}^{a}$ in the next section, where the general case is studied. Despite this fact, this section provides with clarity the basic ingredient of the IGF-COPI approach to the desired hierarchical EOM.

The hierarchy construction starts with the time derivative on the propagator $\mathcal{U}$ [Eq. (9)] of primary interest. The time derivative on the action functional parts contributes to the coherent dynamics of $-i \mathcal{L} U$ and thus can be included in the final EOM. We shall show in the following that the hierarchy generation stems from the time derivative on the evolving influence functionals in each tier of the construction.

Consider first the time derivative on the influence functional of primary interest [Eq. (13)]:

$$
\partial_{t} \mathcal{F}[\boldsymbol{\alpha}]=-\left[\sum_{a} \mathcal{Q}_{a}[\boldsymbol{\alpha}(t)] \widetilde{\mathcal{Q}}_{\boldsymbol{a}}(t ;\{\boldsymbol{\alpha}\})\right] \mathcal{F}[\boldsymbol{\alpha}]
$$

We shall hereafter omit the explicit path integral variable dependence whenever it does not cause confusion. As a result, we recast Eq. (17) as

$$
\partial_{t} \mathcal{F}=-i\left[\sum_{a} \mathcal{Q}_{a}\left(-i \widetilde{\mathcal{Q}}_{\boldsymbol{a}}\right)\right] \mathcal{F} \equiv-i \sum_{\boldsymbol{a}} \mathcal{Q}_{a} \mathcal{F}_{\boldsymbol{a}}
$$

The last identity introduces the auxiliary influence functionals (AIF's)

$$
\mathcal{F}_{a} \equiv\left(-i \widetilde{\mathcal{Q}}_{a}\right) \mathcal{F} .
$$

In contrast to $\mathcal{F}$ whose leading term is 1 , the first-tier AIF's $\left\{\mathcal{F}_{a}\right\}$ are of the second order in the system-bath coupling as their leading terms. The hierarchy to be constructed will go naturally with increasing the order of the system-bath coupling.

Consider now the time derivative on the first-tier AIF's [Eq. (19)]:

$$
\partial_{t} \mathcal{F}_{a}=-i\left(\partial_{t} \widetilde{\mathcal{Q}}_{a}\right) \mathcal{F}-i\left[\sum_{b} \mathcal{Q}_{b}\left(-i \widetilde{\mathcal{Q}}_{a}\right)\left(-i \widetilde{\mathcal{Q}}_{b}\right)\right] \mathcal{F} .
$$

The second term, which arises from the derivative on the primary $\mathcal{F}$ [cf. Eq. (18)], introduces now a set of new AIF's,

$$
\mathcal{F}_{a b} \equiv\left(-i \widetilde{\mathcal{Q}}_{a}\right)\left(-i \widetilde{\mathcal{Q}}_{b}\right) \mathcal{F} .
$$

The leading terms in these second-tier AIF's are of the fourth-order system-bath coupling.

The first term of Eq. (20) involves the time derivative on $\widetilde{\mathcal{Q}}_{a}$ [Eq. (10) with Eqs. (11)]. From Eq. (11a),

$$
\partial_{t} \widetilde{Q}_{a}=C_{a}(0) Q_{a^{\prime}}[\alpha(t)]+\tilde{\tilde{Q}}_{a}(t ;\{\alpha\}),
$$

with 


$$
\tilde{\tilde{Q}}_{a}(t ;\{\alpha\}) \equiv \int_{t_{0}}^{t} d \tau \dot{C}_{a}(t-\tau) Q_{a^{\prime}}[\alpha(\tau)]
$$

Apparently, $\tilde{\tilde{Q}}_{a}$ leads to Eq. (20), a nonhierarchy term in general, unless a specific form of $C_{\boldsymbol{a}}(t)$ such as Eq. (16) is considered. In the present case of study, $\tilde{Q}_{a}=-\gamma_{a} \tilde{Q}_{a}$, leading to

$$
\partial_{t} \widetilde{\mathcal{Q}}_{\boldsymbol{a}}=i \mathcal{C}_{\boldsymbol{a}}-\gamma_{\boldsymbol{a}} \widetilde{\mathcal{Q}}_{\boldsymbol{a}} .
$$

Here, [noting that $C_{a}(0)=\eta_{a}$ from Eq. (16)]

$$
\mathcal{C}_{a}=-i\left\{\eta_{a} Q_{a^{\prime}}[\alpha(t)]-\eta_{a}^{*} Q_{a^{\prime}}\left[\alpha^{\prime}(t)\right]\right\},
$$

which depends only on the fixed ending points. Substituting Eqs. (21) and (24) into Eq. (20) leads to

$$
\partial_{t} \mathcal{F}_{a}=\mathcal{C}_{a} \mathcal{F}-\gamma_{a} \mathcal{F}_{\boldsymbol{a}}-i \sum_{\boldsymbol{b}} \mathcal{Q}_{b} \mathcal{F}_{\boldsymbol{a b}}
$$

We are now in the position to complete the IGF-COPI approach to the hierachical EOM for the multimode DrudeDebye dissipation of Eq. (16). First of all, we notice that due to the mathematical group nature implied in Eqs. (18) and (24), $\left(-i \widetilde{\mathcal{Q}}_{a}\right)$ constitutes the single influence generating functional for each pair of the Drude-Debye modes. The AIF's involved can generically be expressed as [cf. Eqs. (19) and (21)]

$$
\mathcal{F}_{\mathrm{n}} \equiv\left\{\prod_{a}\left(-i \widetilde{\mathcal{Q}}_{a}\right)^{n_{a}}\right\} \mathcal{F}
$$

Here, $\mathrm{n}=\left(n_{a}, n_{b}, \ldots\right)$ consists of a set of non-negative integers. Denote also the index set $\mathrm{n}_{a}^{ \pm} \equiv\left(n_{a} \pm 1, n_{b}, \ldots\right)$, which deviates from $\mathrm{n}$ only by changing the specified $n_{a}$ to $n_{a} \pm 1$. Note that the total number of non-negative integers in the index set $\mathrm{n}$ is the same as that of the nonzero system-bath coupling mode pairs. The time derivative of $\mathcal{F}_{\mathrm{n}}$ can be carried out by using Eq. (24) and the first identity of Eq. (18), resulting in

$$
\partial_{t} \mathcal{F}_{\mathrm{n}}=-\left(\sum_{a} n_{a} \gamma_{a}\right) \mathcal{F}_{\mathrm{n}}+\sum_{a}\left(n_{a} \mathcal{C}_{a} \mathcal{F}_{\mathrm{n}_{a}^{-}}-i \mathcal{Q}_{a} \mathcal{F}_{\mathrm{n}_{a}^{+}}\right) .
$$

Define now the auxiliary propagators by [cf. Eq. (9)]

$$
\mathcal{U}_{\mathrm{n}}\left(\boldsymbol{\alpha}, t ; \boldsymbol{\alpha}_{0}, t_{0}\right) \equiv \int_{\boldsymbol{\alpha}_{0}}^{\boldsymbol{\alpha}} \mathcal{D} \boldsymbol{\alpha} e^{i S[\alpha]} \mathcal{F}_{\mathrm{n}}[\boldsymbol{\alpha}] e^{-i S\left[\alpha^{\prime}\right]},
$$

which also define the related auxiliary density operators,

$$
\rho_{\mathrm{n}}(t) \equiv \mathcal{U}_{\mathrm{n}}\left(t, t_{0}\right) \rho\left(t_{0}\right) .
$$

Equations (28) can then be recast into

$$
\dot{\rho}_{\mathrm{n}}=-\left(i \mathcal{L}+\sum_{a} n_{a} \gamma_{a}\right) \rho_{\mathrm{n}}+\sum_{a}\left(n_{a} \mathcal{C}_{a} \rho_{\mathrm{n}_{a}^{-}}-i \mathcal{Q}_{a} \rho_{\mathrm{n}_{a}^{+}}\right) .
$$

The $\mathcal{Q}_{a}$ and $\mathcal{C}_{a}$ involved, which in Eqs. (28) were given by Eqs. (12) and (25), respectively, at the fixed ending points of $\boldsymbol{\alpha}(t)=\boldsymbol{\alpha}$ in the path integral representation, are now defined at the operator level by Eq. (2) and

$$
\mathcal{C}_{a} \hat{O}=-i\left(\eta_{a} Q_{a^{\prime}} \hat{O}-\eta_{a}^{*} \hat{O} Q_{a^{\prime}}\right) .
$$

Each of the summations on the RHS of Eqs. (31) runs over all nonzero coupling mode pairs $\boldsymbol{a}=\left(a a^{\prime}\right)$. The initial conditions of Eqs. (31) and the methods of infinite hierarchy truncation will be discussed later together with the general dissipation systems; see comments after Eqs. (50) and in Sec. V.

Equations (31) generalize the previous work on singlemode Drude-Debye dissipation [17,19-24]. It is noticed that at the second or higher tier, which is of the fourth or higher order in the system-bath coupling, contributions from different dissipative modes are no longer just additive. Some interesting phenomena such as cotunneling [34] and codissipation (e.g., cooperative $T_{2}$-decoherence and $T_{1}$-relaxation processes) will therefore be anticipated and investigated elsewhere.

\section{HIERARCHICAL EQUATIONS OF MOTION: GENERAL NON-MARKOVIAN DISSIPATION}

\section{A. Non-Markovian bath via parametrization}

It is evident now that the construction of hierarchical EOM for a general dissipative system should involve a proper parametrization scheme for $C_{a a^{\prime}}(t)$. It is required by the IGF-COPI structure that all $\partial_{t} \widetilde{Q}_{a}$ terms involved be contained within the hierarchy; cf. Eqs. (22)-(24) and comments there. On the other hand, relation (6), or more precisely the fluctuation-dissipation theorem (FDT), should also be observed.

In this work, we adopt a FDT-preserved parametrization scheme, in which the bath correlation functions $C_{a}(t)$ for a general system at any temperature are completely characterized by a set of real parameters; see details in Appendix A. In particular, the parameters $\left\{\gamma_{\mathrm{D}}^{a}, \gamma_{k}^{a}, \omega_{k}^{a} ; k=0, \ldots, K\right\}$, which will explicitly enter the final EOM, together with the Matsubara frequencies $\left\{\check{\gamma}_{m} \equiv 2 \pi m / \beta ; m=1, \ldots, M\right\}$, are all positive, except $\omega_{0}^{a} \equiv 0$. For the latter use, we denote also $\omega_{k}^{\prime a}$ $\equiv \delta_{k 0} \gamma_{0}^{a}+\omega_{k}^{a}$.

The final FDT-preserved bath correlation functions assume the following form [cf. Eq. (A4) where $M \rightarrow \infty$ ]:

$$
C_{\boldsymbol{a}}(t>0)=\eta_{\mathrm{D}}^{a} e^{-\gamma_{\mathrm{D}}^{a} t}+\sum_{j=0}^{2 K+1} \eta_{j}^{a} \phi_{j}^{a}(t)+\sum_{m=1}^{M} \check{\eta}_{m}^{a} e^{-\check{\gamma}_{m} t} .
$$

Here, $\check{\gamma}_{m}=2 \pi m / \beta$ and

$$
\begin{gathered}
\phi_{2 k}^{a}(t) \equiv \cos \left(\omega_{k}^{a} t\right) \exp \left(-\gamma_{k}^{a} t\right), \\
\phi_{2 k+1}^{a}(t) \equiv\left[\delta_{k 0} \gamma_{0}^{a} t+\sin \left(\omega_{k}^{a} t\right)\right] \exp \left(-\gamma_{k}^{a} t\right) .
\end{gathered}
$$

The $\eta$ coefficients in Eq. (33) are all complex in general due to the FDT, except those $\left\{\check{\eta}_{m}^{a}\right\}$ arising from the Matsubara contribution are real in a canonical bath ensemble; see Eqs. (A6)-(A9).

Note that $\phi_{0}^{a}(t)=e^{-\gamma_{0}^{a} t}$ and $\phi_{1}^{a}(t)=\gamma_{0}^{a} t e^{-\gamma_{0}^{a} t}$, as $\omega_{0}^{a} \equiv 0$. Also (noting $\omega_{k}^{\prime a} \equiv \delta_{k 0} \gamma_{0}^{a}+\omega_{k}^{a}$ )

$$
\partial_{t} \phi_{2 k}^{a}(t)=-\gamma_{k}^{a} \phi_{2 k}^{a}(t)-\omega_{k}^{a} \phi_{2 k+1}^{a}(t),
$$




$$
\partial_{t} \phi_{2 k+1}^{a}(t)=\omega_{k}^{\prime a} \phi_{2 k}^{a}(t)-\gamma_{k}^{a} \phi_{2 k+1}^{a}(t) .
$$

The above closed relations will be used in the following IGF-COPI construction of hierarchical EOM.

In principle, the parametrized $C_{a}$ in Eq. (33) can be exact for arbitrary dissipation if $K$ and $M$ in Eq. (33) are large enough. The hierarchical EOM to be developed in the rest of this section will also be exact, but its size grows as a power law. The exact evaluation of complex dissipation would rapidly become extremely tedious. We will come back to this issue on how to incorporate the residue correction, due to the small difference between the exact $C_{\boldsymbol{a}}(t)$ (including the zerotemperature case) and the practically used ones, into the final theory in Sec. V.

\section{B. Hierarchical construction: Influence generating functionals}

Note that $\tilde{Q}_{a}$ [Eq. (11)] appears additive with respect to the individual components of $C_{a}$. The dissipation functional $\mathcal{R}$ [Eq. (13b) with Eq. (10)] will also be additive. Moreover, in comparison with the first two terms in Eq. (33), the Matsubara term possesses the special properties of its preexponential factors $\check{\eta}_{m}^{a}$ being real and its time constants $\check{\gamma}_{m}$ being dissipation-mode independent. As a result, the Matsubara contributions to $\mathcal{R}$ [Eq. (13b)] can have the summation over $a^{\prime}$ performed in the following construction of the hierarchical EOM formalism.

To proceed, let us denote $\left[\phi_{\mathrm{D}}^{a}(t) \equiv \exp \left(-\gamma_{\mathrm{D}}^{a} t\right)\right]$

$$
\widetilde{Q}_{j}^{a}(t ;\{\alpha\}) \equiv \int_{t_{0}}^{t} d \tau \phi_{j}^{a}(t-\tau) Q_{a^{\prime}}[\alpha(\tau)]
$$

and

$$
\check{Q}_{m}^{a}(t ;\{\alpha\}) \equiv \sum_{a^{\prime}} \check{\check{\eta}}_{m}^{a} \int_{t_{0}}^{t} d \tau e^{-\check{\gamma}_{m}(t-\tau)} Q_{a^{\prime}}[\alpha(\tau)]
$$

The corresponding composite $\widetilde{\mathcal{Q}}$ functionals that specify the dissipation functional [cf. Eq. (13b)] are denoted as

$$
\begin{gathered}
X_{k}^{a}(t ;\{\boldsymbol{\alpha}\}) \equiv-i\left(\eta_{2 k}^{a} \widetilde{Q}_{2 k}^{a}-\eta_{2 k}^{a^{*}} \widetilde{Q}_{2 k}^{\prime a}\right), \\
Y_{k}^{a}(t ;\{\boldsymbol{\alpha}\}) \equiv-i\left(\eta_{2 k+1}^{a} \widetilde{Q}_{2 k+1}^{a}-\eta_{2 k+1}^{a^{*}} \widetilde{Q}_{2 k+1}^{\prime a}\right), \\
Z_{\mathrm{D}}^{a}(t ;\{\boldsymbol{\alpha}\}) \equiv-i\left(\eta_{\mathrm{D}}^{a} \widetilde{Q}_{\mathrm{D}}^{a}-\eta_{\mathrm{D}}^{a^{*}} \widetilde{Q}_{\mathrm{D}}^{\prime a}\right),
\end{gathered}
$$

and [noting that $\check{\eta}_{m}^{a}$ is real; see Eq. (A9)]

$$
\check{Z}_{m}^{a}(t ;\{\boldsymbol{\alpha}\}) \equiv-i\left(\check{Q}_{m}^{a}-\check{Q}_{m}^{\prime a}\right) .
$$

Included in each of the equations is also the factor of $(-i)$ for the sake of bookkeeping; e.g., $Z_{\mathrm{D}}^{a}$ amounts to the $-i \widetilde{\mathcal{Q}}_{a}$ in Sec. III.

The dissipative functional $\mathcal{R}$, by which

$$
\partial_{t} \mathcal{F}=-\mathcal{R} \mathcal{F},
$$

reads now [cf. Eq. (13)]

$$
\mathcal{R}=i \sum_{a} \mathcal{Q}_{a} Z_{\mathrm{D}}^{a}+i \sum_{a, k} \mathcal{Q}_{a}\left(X_{k}^{a}+Y_{k}^{a}\right)+i \sum_{a, m} \mathcal{Q}_{a} \check{Z}_{m}^{a}
$$

Apparently, all composite $\widetilde{\mathcal{Q}}$ functionals, Eqs. (38), are influence generating functionals; they are, however, not completed.

The crucial step in the IGF-COPI construction of hierarchical EOM is the time derivatives on these composite $\widetilde{\mathcal{Q}}$ functionals (cf. Sec. III). Unlike the $Z$ functionals for the Drude and Matsubara components, the time derivatives of the $X$ and $Y$ functionals are closed together with two additional noncomposite $\widetilde{\mathcal{Q}}$ functionals [cf. Eq. (35)]:

$$
\begin{aligned}
& \bar{X}_{k}^{a}(t ;\{\boldsymbol{\alpha}\}) \equiv-i\left(\eta_{2 k}^{a} \widetilde{Q}_{2 k+1}^{a}-\eta_{2 k}^{a^{*}} \widetilde{Q}_{2 k+1}^{\prime a}\right), \\
& \bar{Y}_{k}^{a}(t ;\{\boldsymbol{\alpha}\}) \equiv-i\left(\eta_{2 k+1}^{a} \widetilde{Q}_{2 k}^{a}-\eta_{2 k+1}^{a^{*}} \widetilde{Q}_{2 k}^{\prime a}\right) .
\end{aligned}
$$

The time derivatives of all $\widetilde{\mathcal{Q}}$ functionals involved are obtained as [cf. Eqs. (35) and (24)]

$$
\begin{gathered}
\partial_{t} X_{k}^{a}=\mathcal{A}_{k}^{a}-\gamma_{k}^{a} X_{k}^{a}-\omega_{k}^{a} \bar{X}_{k}^{a}, \\
\partial_{t} \bar{X}_{k}^{a}=\omega_{k}^{\prime a} X_{k}^{a}-\gamma_{k}^{a} \bar{X}_{k}^{a}, \\
\partial_{t} \bar{Y}_{k}^{a}=\mathcal{B}_{k}^{a}-\gamma_{k}^{a} \bar{Y}_{k}^{a}-\omega_{k}^{a} Y_{k}^{a}, \\
\partial_{t} Y_{k}^{a}=\omega_{k}^{\prime a} \bar{Y}_{k}^{a}-\gamma_{k}^{a} Y_{k}^{a},
\end{gathered}
$$

and

$$
\partial_{t} Z_{\mathrm{D}}^{a}=\mathcal{C}_{\mathrm{D}}^{a}-\gamma_{\mathrm{D}}^{a} Z_{\mathrm{D}}^{a}, \quad \partial_{t} \check{Z}_{m}^{a}=\check{\mathcal{C}}_{m}^{a}-\check{\gamma}_{m} \check{Z}_{m}^{a}
$$

Here

$$
\begin{gathered}
\mathcal{A}_{k}^{a} \equiv-i\left(\eta_{2 k}^{a} Q_{a^{\prime}}-\eta_{2 k}^{a^{*}} Q_{a^{\prime}}^{\prime}\right), \\
\mathcal{B}_{k}^{a} \equiv-i\left(\eta_{2 k+1}^{a} Q_{a^{\prime}}-\eta_{2 k+1}^{a^{*}} Q_{a^{\prime}}^{\prime}\right), \\
\mathcal{C}_{\mathrm{D}}^{a} \equiv-i\left(\eta_{\mathrm{D}}^{a} Q_{a^{\prime}}-\eta_{\mathrm{D}}^{a^{*}} Q_{a^{\prime}}^{\prime}\right), \\
\check{\mathcal{C}}_{m}^{a} \equiv-i \sum_{a^{\prime}} \check{\eta}_{m}^{a}\left(Q_{a^{\prime}}-Q_{a^{\prime}}^{\prime}\right) .
\end{gathered}
$$

Note that Eq. (42c) is identical to Eq. (25). In writing Eq. (42d), we have used the property that $\check{\eta}_{m}^{a}$ is real; see Eq. (A9).

The above six $(X Y Z)$ functionals constitute now a complete set of IGF's. The general expression for the AIF's in the hierarchy are then obtained as [cf. Eq. (27) and its comment above]

$$
\begin{aligned}
\mathcal{F}_{\mathrm{n}}= & \left\{\prod_{\boldsymbol{a}, k}\left[\left(X_{k}^{a}\right)^{a_{2 k}^{a}}\left(Y_{k}^{a}\right)^{n_{2 k+1}^{a}}\left(\bar{X}_{k}^{a}\right)^{\bar{n}_{2 k}^{a}}\left(\bar{Y}_{k}^{a}\right)^{\bar{n}_{2 k+1}^{a}}\right]\right. \\
& \left.\times \prod_{a}\left(Z_{\mathrm{D}}^{a}\right)^{n_{\mathrm{D}}^{a}} \prod_{a, m}\left(\check{Z}_{m}^{a}\right)^{\check{n}_{m}^{a}}\right\} \mathcal{F} .
\end{aligned}
$$

The index in $\mathcal{F}_{\mathrm{n}}$ is specified by the involving non-negative integers 


$$
\mathrm{n} \equiv\left(n_{j}^{a}, \bar{n}_{j}^{a}, n_{\mathrm{D}}^{a}, \check{n}_{m}^{a} ; j=0, \ldots, 2 K+1 ; m=1, \ldots, M\right)
$$

Therefore, the total number of non-negative integers in the index set $\mathrm{n}$ is $4(K+1) p+p+M q$, where $q$ denotes the number of dissipative modes and $p \leqslant q^{2}$ the number of nonzero dissipative mode pairs.

\section{Hierarchical equations of motion}

The IGF-COPI approach to the hierarchical EOM can now be completed by taking the time derivative on $\mathcal{F}_{\mathrm{n}}$ [Eq. (43) with Eqs. (39) and (41)]. The final results read in terms of the auxiliary density operators as [cf. Eqs. (29) and (30)]

$$
\dot{\rho}_{\mathrm{n}}=-\left(i \mathcal{L}+\gamma_{\mathrm{n}}\right) \rho_{\mathrm{n}}+\rho_{\mathrm{n}}^{\{\leftrightarrows\}}+\rho_{\mathrm{n}}^{\{-\}}+\rho_{\mathrm{n}}^{\{+\}} .
$$

The $\gamma$ term in Eqs. (45) arises from the damping terms of Eqs. (41). The resulting damping constant is given by

$$
\gamma_{\mathrm{n}} \equiv \sum_{\boldsymbol{a}, k}\left(n_{2 k}^{\boldsymbol{a}}+\bar{n}_{2 k}^{a}+n_{2 k+1}^{\boldsymbol{a}}+\bar{n}_{2 k+1}^{a}\right) \gamma_{k}^{a}+\sum_{\boldsymbol{a}} n_{\mathrm{D}}^{\boldsymbol{a}} \gamma_{\mathrm{D}}^{\boldsymbol{a}}+\sum_{a, m} \check{n}_{m}^{a} \check{\gamma}_{m}
$$

The second term in Eq. (45) stems from the (off-diagonal) swap terms of Eqs. (41a)-(41d). It reads

$$
\begin{aligned}
\rho_{\mathrm{n}}^{\{\leftrightarrows\}}= & -\sum_{\boldsymbol{a}, k} \omega_{k}^{\boldsymbol{a}}\left(n_{2 k}^{\boldsymbol{a}} \rho_{\mathrm{n}_{\boldsymbol{a}, 2 k}}+\bar{n}_{2 k+1}^{a} \rho_{\mathrm{n}_{\boldsymbol{a}, 2 k+1}^{\leftarrow}}\right) \\
& +\sum_{\boldsymbol{a}, k} \omega_{k}^{\prime a}\left(\bar{n}_{2 k}^{a} \rho_{\mathrm{n}_{\boldsymbol{a}, 2 k}^{\leftarrow}}+n_{2 k+1}^{a} \rho_{\mathrm{n}_{\boldsymbol{a}, 2 k+1}}\right) .
\end{aligned}
$$

The index-set $\mathrm{n}_{\vec{a}, j}$ differs from $\mathrm{n}$ of Eq. (44) only by $\left(n_{j}^{a}, \bar{n}_{j}^{a}\right) \rightarrow\left(n_{j}^{a}-1, \bar{n}_{j}^{a}+1\right), \quad$ while $\quad \mathrm{n}_{a, j}^{\leftarrow} \quad$ by $\quad\left(n_{j}^{a}+1, \bar{n}_{j}^{a}-1\right)$ $\leftarrow\left(n_{j}^{a}, \bar{n}_{j}^{a}\right)$, at the specified $(\boldsymbol{a}, j)$.

The third term in Eq. $(45)$ stems from the $(\mathcal{A}, \mathcal{B}, \mathcal{C})$ terms of Eqs. (41), while the last term is from Eq. (39). They are the hierarchy-down and hierarchy-up contributions, respectively, and given by

$$
\begin{aligned}
\rho_{\mathrm{n}}^{\{-\}}= & \sum_{\boldsymbol{a}, k}\left(n_{2 k}^{\boldsymbol{a}} \mathcal{A}_{k}^{\boldsymbol{a}} \rho_{\mathrm{n}_{\boldsymbol{a}, 2 k}^{-}}+\bar{n}_{2 k+1}^{a} \mathcal{B}_{k}^{a} \rho_{\overline{\mathrm{n}}-{ }_{\boldsymbol{a}, 2 k+1}^{-}}\right) \\
& +\sum_{\boldsymbol{a}} n_{\mathrm{D}}^{\boldsymbol{a}} \mathcal{C}_{\mathrm{D}}^{\boldsymbol{a}} \rho_{\mathrm{n}_{\boldsymbol{a}, \mathrm{D}}^{-}}+\sum_{a, m} \check{n}_{m}^{a} \check{\mathcal{C}}_{m}^{a} \rho_{\check{\mathrm{n}}_{a, m}^{-}}
\end{aligned}
$$

and $(j=\mathrm{D}, 0,1, \cdots, 2 K+1)$

$$
\rho_{\mathrm{n}}^{\{+\}} \equiv-\mathcal{R} \rho_{\mathrm{n}}=-i \sum_{a, j} \mathcal{Q}_{a} \rho_{\mathrm{n}_{a, j}^{+}}-i \sum_{a, m} \mathcal{Q}_{a} \rho_{\check{\mathrm{n}}_{a, m}^{+}} .
$$

The index set $\mathrm{n}_{a, j}^{ \pm}\left(\overline{\mathrm{n}}_{a, j}^{-}\right.$or $\left.\mathrm{n}_{a, m}^{ \pm}\right)$differs from $\mathrm{n}$ only by changing the specified $n_{j}^{a}\left(\bar{n}_{j}^{a}\right.$ or $\left.\check{n}_{m}^{a}\right)$ to $n_{j}^{a} \pm 1\left(\bar{n}_{j}^{a}-1\right.$ or $\left.\check{n}_{m}^{a} \pm 1\right)$. Note that the $\left(\overline{\mathrm{n}}_{a, j}^{+}\right)$th-auxiliary-reduced density operators are not generated from Eq. (49), since $\bar{X}_{k}$ and $\bar{Y}_{k}$ do not appear in the dissipation functional $\mathcal{R}$ [Eq. (39)]; they are rather generated from the EOM for the $\left(\mathrm{n}_{a, j}^{+}\right)$th-auxiliary-reduced density operators via the involving swap $\{\leftrightarrows\}$-terms there [cf. Eq. (47)].

In Eq. (48), $\mathcal{A}_{k}^{a}, \mathcal{B}_{k}^{a}, \mathcal{C}_{\mathrm{D}}^{a}$, and $\check{\mathcal{C}}_{m}^{a}$ denote the reduced Liouville-space operator counterparts of Eqs. (42) [cf. the comments above Eq. (32)]:

$$
\mathcal{A}_{k}^{a} \hat{O}=-i\left(\eta_{2 k}^{a} Q_{a^{\prime}} \hat{O}-\eta_{2 k}^{a^{*}} \hat{O} Q_{a^{\prime}}\right),
$$

$$
\begin{gathered}
\mathcal{B}_{k}^{a} \hat{O}=-i\left(\eta_{2 k+1}^{a} Q_{a^{\prime}} \hat{O}-\eta_{2 k+1}^{a^{*}} \hat{O} Q_{a^{\prime}}\right), \\
\mathcal{C}_{\mathrm{D}}^{a} \hat{O}=-i\left(\eta_{\mathrm{D}}^{a} Q_{a^{\prime}} \hat{O}-\eta_{\mathrm{D}}^{a^{*}} \hat{O} Q_{a^{\prime}}\right), \\
\check{\mathcal{C}}_{m}^{a} \hat{O}=-i \sum_{a^{\prime}} \check{\eta}_{m}^{a}\left[Q_{a^{\prime}}, \hat{O}\right] .
\end{gathered}
$$

The reduced Liouville-space operator $\mathcal{Q}_{a}$ involved in Eq. (49) was given by Eq. (2).

The initial conditions to Eqs. (45) are $\rho_{\mathrm{n}}\left(t_{0}\right)=\rho\left(t_{0}\right) \delta_{\mathrm{n} 0}$, as inferred from their definitions, and $\rho_{0}(t)=\rho(t)$ is the reduced density operator of primary interest. Note that when the initial time $t_{0} \rightarrow-\infty$, the established hierarchical EOM formalism imposes no approximation. The initial conditions are, however, $\dot{\rho}_{\mathrm{n}}\left(t_{0}\right)=0$, where $t_{0}$ can be any time before applying the external time-dependent fields. The pulse-field-induced dynamics will then be evaluated via Eq. (45).

The hierarchical EOM, Eqs. (45)-(50), are exact for a general dissipation system that involves the parametrized bath correlation functions of Eqs. (33). The residue effect due to the small difference between the exact and parametrized $C_{\boldsymbol{a}}(t)$ on the final formalism will be carried out together with the hierarchy truncation via the principle of residue correction in the next section.

It is noted that with a proper rearrangement, Eqs. (45) can be recast into the standard tridiagonal coupling form; see Appendix B. Included there is also a variation of the above theory, expressed in terms of the hierarchical Green's functions and their related memory kernels and continuedfraction formalism.

\section{TRUNCATION AND THE PRINCIPLE OF RESIDUE CORRECTION}

\section{A. Principle of residue correction}

To complete the EOM formalism, the infinite hierarchy in Eqs. (45)-(50) should be truncated at a certain level-say, the $\left(N_{\text {trun }}\right)$ th tier. The simplest way is to set all $\left\{\rho_{n}\right\}$ of the higher tiers to be zero. The resulting $\rho_{0}(t)=\rho(t)$ of primary interest will be exact up to the $\left(2 N_{\text {trun }}\right)$ th order in the systembath coupling. Other truncation schemes, related to different ways to a partial account of the higher-order effect, have also been proposed [17,19-22].

Here, we present the principle of residue correction, which itself is formally exact. It is applied not just to the truncation, but also to a recovery of the residue effect, due to the difference between the exact and parametrized $C_{\boldsymbol{a}}(t)$ of Eq. (33), at all levels of hierarchy. The hierarchy truncation concerning only that at the anchor level will be treated in the next subsection.

The principle of residue correction related to the finite difference between the exact and parametrized ones,

$$
\delta C_{a}(t) \equiv C_{a}^{\mathrm{exa}}(t)-C_{a}(t),
$$

arises from the observation that the dissipation functional is additive [cf. Eq. (13b) with Eqs. (10) and (11)]: 


$$
\mathcal{R}^{\operatorname{ex}}[t ;\{\boldsymbol{\alpha}\}]=\mathcal{R}[t ;\{\boldsymbol{\alpha}\}]+\delta \mathcal{R}[t ;\{\boldsymbol{\alpha}\}] .
$$

Here,

$$
\delta \mathcal{R}[t ;\{\boldsymbol{\alpha}\}]=\sum_{a} \mathcal{Q}_{a}[\boldsymbol{\alpha}] \delta \widetilde{\mathcal{Q}}_{a}(t ;\{\boldsymbol{\alpha}\}),
$$

with $\delta \widetilde{\mathcal{Q}}_{a}$ the same as Eq. (10), but associating with $\delta C_{a}(t)$; i.e., [cf. Eq. (11a)],

$$
\delta \widetilde{Q}_{a}(t ;\{\alpha\})=\int_{t_{0}}^{t} d \tau \delta C_{a}(t-\tau) Q_{a^{\prime}}[\alpha(\tau)] .
$$

The resulting exact influence functional of primary interest reads now $\mathcal{F}^{\operatorname{ex}}[\boldsymbol{\alpha}]=\mathcal{F}_{\text {resi }}[\boldsymbol{\alpha}] \mathcal{F}[\boldsymbol{\alpha}]$, with $\partial_{t} \mathcal{F}_{\text {resi }}=-\delta \mathcal{R} \mathcal{F}_{\text {resi }}$. The AIF's defined in Eq. (43) for the construction of the hierarchical EOM should now be replaced by

$$
\mathcal{F}_{\mathrm{n}}^{\text {exa }} \equiv \mathcal{F}_{\mathrm{n}} \mathcal{F}_{\text {resi }} \text {. }
$$

Its time derivative reads

$$
\partial_{t} \mathcal{F}_{\mathrm{n}}^{\text {exa }}=\left(\partial_{t} \mathcal{F}_{\mathrm{n}}\right) \mathcal{F}_{\text {resi }}-\delta \mathcal{R} \mathcal{F}_{\mathrm{n}}^{\text {exa }} .
$$

The EOM for the corresponding exact $\rho_{\mathrm{n}}$ is then obtained as [cf. Eqs. (45)-(50)]

$$
\dot{\rho}_{\mathrm{n}}=-\left[i \mathcal{L}+\delta \mathcal{R}(t)+\gamma_{\mathrm{n}}\right] \rho_{\mathrm{n}}+\rho_{\mathrm{n}}^{\{\leftrightarrows\}}+\rho_{\mathrm{n}}^{\{-\}}+\rho_{\mathrm{n}}^{\{+\}} .
$$

The residue correction due to $\delta C_{a}(t)$ [Eq. (51)] is thus global; the resulting $\delta \mathcal{R}(t)$ [Eq. (53)] modifies the individual hierarchical EOM at all levels.

Apparently, $\delta \mathcal{R}$ of Eq. (53) and $\mathcal{R}$ of Eq. (13b) are of the same mathematical structure. If their common operator-level expression were known and implementable readily without approximation, Eq. (14b) would be used directly, without invoking the hierarchical EOM at all. However, it is only possible for special cases, such as the pure-dephasing limit (i.e., the case of $\left[H, Q_{a}\right]=0$ ) or the driven Brownian oscillator system [16].

The key idea behind Eq. (57) for general non-Markovian dissipation is as follows. The total $C_{a}^{\text {exa }}$ is partitioned into two parts. One is the parametrized $C_{a}$, which carries most of the non-Markovian coupling strength and is expressed in the form of Eq. (33). Another is the residue $\delta C_{\boldsymbol{a}}$, which is assumed in the weak-interaction regime. The strong dissipation due to parametrized $C_{a}$ is treated via the hierarchical EOM approach developed in Sec. IV without approximation. In principle, the residue $\delta C_{a}$ can be zero if $K$ and $M$ for the parametrized $C_{\boldsymbol{a}}$ in Eq. (33) are large enough. However, the size of hierarchical EOM grows as a power law as $K$ and $M$ increase; the exact evaluation of complex dissipation would rapidly become extremely tedious if not impossible. Therefore, it is a practical trade-off to have a nonzero but weak $\delta C_{a}$, as long as its induced global residue $\delta \mathcal{R}$ can be accurately described with a certain perturbative or nonperturbative formulation at the operator level.

Let us start with the simplest one, the Markovian-residue limit, in which Eq. (54) reduces to

$$
\delta \tilde{Q}_{a} \approx \delta \bar{C}_{a}(t) Q_{a^{\prime}}[\alpha(t)]
$$

with

$$
\delta \bar{C}_{a}(t)=\int_{t_{0}}^{t} d \tau \delta C_{a}(t-\tau) .
$$

Note that the system variable $Q_{a^{\prime}}$ in Eq. (58) is now represented at the ending time $t$ of the path integral at which $\alpha(t)=\alpha$ and $\alpha^{\prime}(t)=\alpha^{\prime}$ are fixed. As a result, Eq. (53) for the Markovian-residue dissipation can be expressed in the operator level as

$$
\delta \mathcal{R}(t) \hat{O}=\sum_{a}\left[Q_{a}, \delta \bar{C}_{a}(t) Q_{a^{\prime}} \hat{O}-\left[\delta \bar{C}_{a}(t)\right]^{*} \hat{O} Q_{a^{\prime}}\right] .
$$

When $t \rightarrow \infty$, the above equation reduces to the Matsubararesidue or finite-temperature correction proposed by Ishizaki and Tanimura [21,22]. Their hierarchical EOM is the singlemode Drude dissipation version of Eq. (57).

The principle of residue correction is right rooted at the fact that $\delta \mathcal{R}$ [Eq. (53)] is of the same mathematical structure as time-local dissipation functional $\mathcal{R}(t)$ [Eq. (13b)]. As results, various well-established approximation schemes can be exploited for the superoperator $\delta \mathcal{R}$, as it describes weak residue dissipation. The most celebrated scheme may be the second-order time-local expression [13-16]

$$
\delta \mathcal{R}(t) \hat{O} \approx \sum_{a}\left[Q_{a}, \delta \widetilde{Q}_{a}^{(2)}(t) \hat{O}-\hat{O}\left[\delta \widetilde{Q}_{a}^{(2)}(t)\right]^{\dagger}\right],
$$

where

$$
\delta \widetilde{Q}_{a}^{(2)}=\int_{t_{0}}^{t} d \tau \delta C_{\boldsymbol{a}}(t-\tau) e^{-i \mathcal{L}(t-\tau)} Q_{a^{\prime}}
$$

The dissipation-free propagator (assuming also a timeindependent system Hamiltonian for simplicity) is used here to connect $Q_{a^{\prime}}[\alpha(\tau)]$ in Eq. (54) to its value at the fixed ending path point of $\alpha(t)=\alpha$. The above operator-level expressions are thus obtained [17]. In fact, Eqs. (61) constitute the unified Bloch-Redfield-Fokker-Planck equation $[14,16]$.

Nonperturbative approximation schemes can also be applied to the evaluation of the global residue-induced $\delta \mathcal{R}(t)$. These include the noninteracting blip approximation or its variations $[4,8,12]$, the self-energy augmented methods [35], and the use of the exactly solvable driven Brownian oscillator model [16] to mimic the anharmonic system of interest.

\section{B. Hierarchy truncation via the principle of residue correction}

Consider now the hierarchy truncation and its related issues. Apparently, the collection of anchors $\left\{\rho_{\mathrm{N}}\right\}$ should be properly specified. The hierarchy tier-up $\rho_{\mathrm{N}}^{\{+\}}$associated with an anchor $\rho_{\mathrm{N}}$ contains at least one component that goes beyond the desired anchoring confinement and, thus, is subject to truncation.

The principle of residue correction is applied here by recognizing that the tier-up components can be recast as [cf. Eqs. (43)]

$$
\begin{aligned}
& \rho_{\mathrm{N}_{a, 2 k}^{+}}+\rho_{\mathrm{N}_{a, 2 k+1}^{+}}=\left(X_{k}^{a}+Y_{k}^{a}\right) \rho_{\mathrm{N}}, \\
& \rho_{\mathrm{N}_{a, \mathrm{D}}^{+}}=Z_{\mathrm{D}}^{a} \rho_{\mathrm{N}}, \quad \rho_{\mathrm{N}_{a, m}^{+}}=\check{Z}_{m}^{a} \rho_{\mathrm{N}} .
\end{aligned}
$$


The $(2 k)$ th and $(2 k+1)$ th components for each dissipative mode pair $\boldsymbol{a}$ are grouped together for the reasons that they arise from the same term of the parametrized spectral density function and they carry the same strength; cf. Appendix A. Therefore, they shall be treated equally as the truncation is concerned.

The anchoring indices can now be specified for the individual constituents of the interaction bath correlation functions $C_{\boldsymbol{a}}(t)$ of Eq. (33) as

$$
N_{\mathrm{D}}^{a}, N_{k}^{a}, \check{N}_{m}^{a}, \quad \text { with } k=0, \ldots, K ; \quad m=1, \ldots, M .
$$

The closed set of hierarchically coupled EOM will then contain those $\rho_{\mathrm{n}}$ whose individual index set consists of the nonnegative integers that are confined within

$$
\begin{gathered}
n_{2 k}^{a}+n_{2 k+1}^{a}+\bar{n}_{2 k}^{a}+\bar{n}_{2 k+1}^{a} \leqslant N_{k}^{a}, \\
n_{\mathrm{D}}^{a} \leqslant N_{\mathrm{D}}^{a}, \quad \check{n}_{m}^{a} \leqslant \check{N}_{m}^{a} .
\end{gathered}
$$

The anchor index set $\mathrm{N}$ in $\rho_{\mathrm{N}}$ can now be defined as those with at least one of the above upper limits being reached. The constraint of Eq. (63a) is consistent with the way of grouping in Eq. (62a). Once the upper limit of Eq. (63a) is reached, the associated tier-up $\rho_{\mathrm{N}_{a, 2 k}^{+}}$and $\rho_{\mathrm{N}_{a, 2 k+1}^{+}}$are both subject to the truncation.

The truncation can now be made based on the formally exact relations of Eqs. (62). The $X_{k}^{a}, Y_{k}^{a}, Z_{\mathrm{D}}^{a}$, and $\check{Z}_{m}^{a}$ involved there are the reduced Liouville-space operators, whose path integral representation counterparts were given by Eqs. (38). Taking Eq. (38a) for $X_{k}^{a}$ for an example, its operator-level form reads in contact with Eq. (62a) as

$$
\rho_{\mathrm{N}_{a}^{+}, 2 k} \equiv X_{k}^{a} \rho_{\mathrm{N}}=-i\left[\eta_{2 k}^{a} \widetilde{Q}_{2 k}^{a}(t) \rho_{\mathrm{N}}-\eta_{2 k}^{a^{*}} \rho_{\mathrm{N}} \widetilde{Q}_{2 k}^{a \dagger}(t)\right] .
$$

If $\rho_{\mathrm{N}_{a, 2 k}^{+}}$goes beyond the closed hierarchy, an approximated expression of $\widetilde{Q}_{2 k}^{a}(t)$, such as [cf. Eq. (61b) and the comments there]

$$
\widetilde{Q}_{2 k}^{a}(t) \approx \int_{t_{0}}^{t} d \tau \phi_{2 k}^{a}(t-\tau) e^{-i \mathcal{L}(t-\tau)} Q_{a^{\prime}},
$$

is adopted locally on the RHS of Eq. (64) to make the truncation. The resulting $\rho_{\mathrm{N}_{a, 2 k}^{+}}$retains the same leading term as the exact one, being of $(2 N+2)$ th order in the specified system-bath coupling strength. Thus, the truncation with a sufficiently large $N$ induces practically no error as far as the dynamics of $\rho(t)=\rho_{0}(t)$ of primary interest is concerned. The construction of closed hierarchical EOM with residue correction is now completed.

\section{Discussions and comments}

We reemphasize here that when the values of $K$ and $M$ in Eq. (33) and the truncation anchor indices in Eq. (63) are set to be sufficiently large, the residue effects on the primarily interested $\rho$ are effectively zero. The global residue correction and the truncation scheme introduced at both the global and local truncation levels in the previous two subsections are made for the purpose of efficient evaluation of the re- duced dynamics of primary interest. For example, one may simply terminate the hierarchical EOM by setting the aforementioned beyond-the-hierarchy $\rho_{\mathrm{N}_{a, 2 k}^{+}}=0$. The resulting $\rho$ of primary interest will be exact up to the $(2 N)$ th order in the specified system-bath coupling strength. The improved truncation as Eq. (64) will lead to $\rho$ exact up to $(2 N+2)$ th order, rather than $(2 N)$ th order. The above two truncation schemes, which represent two different resummations for partially incorporating the higher-order effects on the reduced dynamics of primary interest, shall be of no practical difference when the convergence is reached.

Note that various commonly used forms of the BlochRedfield theory and Fokker-Planck equations can be considered as globally weak (residue) dissipation without invoking the hierarchical EOM at all. They can also be recovered if the second-order truncation scheme of Eq. (65) is applied to the primary tier of $\mathrm{N}=0$. On the other hand, if all second-tier auxiliary reduced density operators are set to be zero, the present hierarchical EOM formalism reduces to the secondorder memory-kernel quantum dissipation theory.

The global residue dissipation $\delta \mathcal{R}(t)$ introduced in the final formalism [Eq. (57)] is also for the purpose of efficiency. Note that the number of the $n$ th-tier auxiliary reduced density operators $\left\{\rho_{n}\right\}$ is $\frac{(n+P-1) !}{n !(P-1) !}$, where $P=4(K+1) p+p$ $+M q$ is the number of the non-negative integers in the index $\mathrm{n}$; see comments after Eq. (44) and in Appendix B before Eq. (B1). The size of the hierarchical EOM increases as a power law as the values of $K$ and $M$ for the parametrized $C_{a}$ of Eq. (33). The residue correction is introduced to reduce the required values of $K$ and $M$, as long as the induced residue weak dissipation $\delta \mathcal{R}(t)$ can be accurately evaluated. In this sense, the final residue-corrected hierarchical EOM formalism remains practically exact. It is worthy to point out that the final formalism is even capable of treating zerotemperature dissipation. At $T=0$, the Matsubara frequencies are all vanished and the exact $C_{a}^{\text {exa }}(t)$ is given by Eq. (A10). However, one can set a certain finite low temperature for the parametrized $C_{a}$ and evaluate residue $\delta C_{a}$-corrected hierarchical EOM dynamics, followed by the convergence test with a lower value of the parametrization temperature.

\section{SUMMARY}

In summary, we have constructed the residue-corrected hierarchical EOM formalism [Eq. (57) with Eqs. (46)-(50)]. The construction consists of two main steps. The first is the IGF-COPI approach to the EOM formalism (Sec. IV), for the parametrized interaction bath correlation functions preserving the fluctuation-dissipation theorem. This step is itself exact, as the FDT-preserved parametrization involved can in principle represent arbitrary interaction bath correlation functions. The second step is the residue correction, concerned with the practical applicability of the present theory to a broad range of systems. The principle of residue correction (Sec. V) is itself exact, rooted in the formal relation between the dissipation functional and the time-local dissipation superoperator; see Sec. II C. The application of this principle to construct the global ( $\delta C$-induced) or the local (truncation- 
induced) residue correction invokes inevitably a certain approximation scheme. However, it can be made in a sufficiently accurate manner, as far as the primarily interested $\rho(t)$ is concerned (cf. Sec. V C). As a result, the residuecorrected hierarchical EOM formalism could be practically used in the study of general quantum dissipation systems interacting with arbitrary bath, arbitrary time-dependent external fields, and at any temperature, including $T=0$; see the last remark stated in Sec. V C.

The hierarchical EOM formalism may be relatively tractable, in comparison with the direct evaluation of the path integral formalism [18]. The memory effect in the quasiadiabatic propagator path integral method is described in terms of the nonlocality of the influence functional [18], while it in the present differential formalism is resolved via a set of linearly coupled time-local auxiliary operators. The exponential-like series expansion of the parametrized $C_{\boldsymbol{a}}(t)$ [Eq. (33)] can be considered as the separation of the time scales, and the resulting $\rho_{\mathrm{n}}$ is associated with the decay constant $\gamma_{n}$. The different truncation anchor indices [Eq. (63)] can therefore be identified to reduce the required number of equations, which otherwise grows exponentially if all $C_{a}$ composites are treated equally. The weak residue correction at the global level provides additional freedom to improve the numerical efficiency of the present EOM theory.

Numerous existing quantum dissipation theories can be recovered from the present formalism. These include the Tanimura's hierarchical EOM for single-mode Drude dissipation $[21,22]$ and the unified Bloch-Redfield-Fokker-Planck formulation [14,16]; see comments after Eqs. (60) and the second paragraph of Sec. V C, respectively. The equivalent hierarchical and continued-fraction Green's functions and memory kernels expressions are also presented; see Appendix B. The application of the continued-fraction Green's function formalism to the two-state electron transfer system, with a single-mode Drude-Debye dissipation in the hightemperature limit, has been carried out recently, resulting in an analytical expression for the nonperturbative rate process $[36,37]$.

Multimode dissipation is physically important. In the weak (second-order) dissipation regime, the effects of different system-bath coupling modes are additive. This simple property is no longer true in the strong-dissipation regime, and it has been shown that the strong dissipation should include also the cases of long-memory system-bath interactions [17]. Cooperative dissipation, similar to cotunneling in quantum transport [34], could be a common phenomenon in reality. The present work is carried out in the bosonic canonical bath case. The extension to the grand canonical bath ensemble cases, including both fermion and boson statistics, will be treated elsewhere [33].

\section{ACKNOWLEDGMENTS}

Support from the NNSF of China (Grants Nos. 50121202, 20403016, and 20533060), Ministry of Education of China (Grant No. NCET-05-0546), and the RGC Hong Kong (Grant No. 604006) is acknowledged.

\section{APPENDIX A: PARAMETRIZATION OF BATH CORRELATION FUNCTIONS}

The fluctuation-dissipation theorem relates the correlation functions $C_{a a^{\prime}}(t)$ and the spectral density functions $J_{a a^{\prime}}(\omega)$ by

$$
C_{a a^{\prime}}(t)=\frac{1}{\pi} \int_{-\infty}^{\infty} d \omega \frac{e^{-i \omega t} J_{a a^{\prime}}(\omega)}{1-e^{-\beta \omega}} .
$$

The spectral density functions $J_{a a^{\prime}}(\omega)$ in a canonical ensemble satisfy in general the symmetry relations

$$
J_{a a^{\prime}}(\omega)=-J_{a^{\prime} a}(-\omega)=J_{a^{\prime} a}^{*}(\omega) .
$$

The FDT leads also to $J_{a a^{\prime}}(0)=0$, the spectrum positivity $J_{a a}(\omega>0) \geqslant 0$, and the Schwarz inequality $J_{a a}(\omega) J_{b b}(\omega)$ $\geqslant\left|J_{a b}(\omega)\right|^{2}$.

We adopt the following form of the extended MeierTannor spectral-density parametrization scheme [16,38], in which (setting $\omega_{0}^{a} \equiv 0$ )

$$
J_{a}(\omega)=\frac{\zeta_{\mathrm{D}}^{a} \omega}{\omega^{2}+\left(\gamma_{\mathrm{D}}^{a}\right)^{2}}+\sum_{k=0}^{K} \frac{\zeta_{k}^{a} \gamma_{k}^{a} \omega+i \bar{\zeta}_{k}^{a} \omega^{2}}{\left|\omega^{2}-\left(\omega_{k}^{a}+i \gamma_{k}^{a}\right)^{2}\right|^{2}} .
$$

All parameters involved are real: $\omega_{k \neq 0}^{a}, \gamma_{k}^{a}$ and $\gamma_{\mathrm{D}}^{a}$ positive as well. The symmetry relations in Eq. (A2) require also (noting that $\left.\bar{\zeta}_{k}^{a a}=0\right)$

$$
\left(\gamma_{\mathrm{D}}^{b a}, \zeta_{\mathrm{D}}^{b a}, \omega_{k}^{b a}, \gamma_{k}^{b a}, \zeta_{k}^{b a}, \bar{\zeta}_{k}^{b a}\right)=\left(\gamma_{\mathrm{D}}^{a b}, \zeta_{\mathrm{D}}^{a b}, \omega_{k}^{a b}, \gamma_{k}^{a b}, \zeta_{k}^{a b},-\bar{\zeta}_{k}^{a b}\right)
$$

The FDT [Eq. (A1)] leads to the interaction bath correlation functions of [cf. Eq. (B4) of Ref. [16]]

$$
C_{\boldsymbol{a}}(t>0)=\eta_{\mathrm{D}}^{a} e^{-\gamma_{\mathrm{D}}^{a} t}+\sum_{j=0}^{2 K+1} \eta_{j}^{a} \phi_{j}^{a}(t)+\sum_{m=1}^{\infty} \check{\eta}_{m}^{a} e^{-\check{\gamma}_{m} t},
$$

with (noting $\left.\omega_{0}^{a} \equiv 0\right)$

$$
\begin{gathered}
\phi_{2 k}^{a}(t) \equiv \cos \left(\omega_{k}^{a} t\right) \exp \left(-\gamma_{k}^{a} t\right), \\
\phi_{2 k+1}^{a}(t) \equiv\left[\delta_{k 0} \gamma_{0}^{a} t+\sin \left(\omega_{k}^{a} t\right)\right] \exp \left(-\gamma_{k}^{a} t\right) .
\end{gathered}
$$

The first term on the RHS of Eq. (A4) arises from the pole of the Drude term in Eq. (A3). The coefficient involved is given by

$$
\eta_{\mathrm{D}}^{a}=\frac{\zeta_{\mathrm{D}}^{a}}{2}\left[\cot \left(\beta \gamma_{\mathrm{D}}^{a} / 2\right)-i\right]
$$

The second term on the RHS of Eq. (A4) arises from the poles of the second term in Eq. (A3). The functions $\phi_{j}(t)$ involved, given by Eqs. (34), are chosen to be real in this work, rather than complex exponential functions used in Ref. [16]. Note that $\phi_{0}^{a}(t)=e^{-\gamma_{0}^{a} t}$ and $\phi_{1}^{a}(t)=\gamma_{0}^{a} t \phi_{0}^{a}(t)$, as inferred from $\omega_{0}^{a} \equiv 0$. The coefficients $\left\{\eta_{j}^{a}\right\}$ involved are complex. Those with even indices are (including $k=0$ at which $\omega_{0}^{a}=0$ ) 


$$
\eta_{2 k}^{a}=\frac{\left(\zeta_{k}^{a}+\bar{\zeta}_{k}^{a}\right) \gamma_{k}^{a} \sinh \left(\beta \omega_{k}^{a}\right)-\bar{\zeta}_{k}^{a} \omega_{k}^{a} \sin \left(\beta \gamma_{k}^{a}\right)}{4 \omega_{k}^{a} \gamma_{k}^{a}\left[\cosh \left(\beta \omega_{k}^{a}\right)-\cos \left(\beta \gamma_{k}^{a}\right)\right]}+i \frac{\bar{\zeta}_{k}^{a}}{4 \gamma_{k}^{a}}
$$

and those with odd indices are

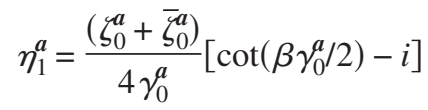

and $(2 k+1=3,5, \ldots)$

$$
\eta_{2 k+1}^{a}=\frac{\bar{\zeta}_{k}^{a} \omega_{k}^{a} \sinh \left(\beta \omega_{k}^{a}\right)+\left(\zeta_{k}^{a}+\bar{\zeta}_{k}^{a}\right) \gamma_{k}^{a} \sin \left(\beta \gamma_{k}^{a}\right)}{4 \omega_{k}^{a} \gamma_{k}^{a}\left[\cosh \left(\beta \omega_{k}^{a}\right)-\cos \left(\beta \gamma_{k}^{a}\right)\right]}-i \frac{\zeta_{k}^{a}+\bar{\zeta}_{k}^{a}}{4 \omega_{k}^{a}} .
$$

The last term on the RHS of Eq. (A4) arises from the Matsubara frequencies,

$$
\check{\gamma}_{m}=2 \pi m / \beta, \quad m=1,2, \ldots,
$$

which constitute poles of the $\left(1-e^{-\beta z}\right)^{-1}$ factor in Eq. (A1). The coefficients involved are

$$
\check{\eta}_{m}^{a} \equiv-i(2 / \beta) J_{\boldsymbol{a}}\left(-i \check{\gamma}_{m}\right)=\left(\check{\eta}_{m}^{a}\right)^{*}, \quad m=1,2, \ldots .
$$

Note that in general, while $J_{a} \equiv J_{a a^{\prime}}(\omega)$ can be complex when $a \neq a^{\prime}$, its analytical continuation to $J_{a}(i \omega)$ is purely imaginary in a canonical ensemble system, as inferred from Eq. (A2). As a result, $\check{\eta}_{m}^{a}$ of Eq. (A9) are all real.

In principle, Eqs. (A4)-(A9) can be exact at an arbitrary finite temperature if $K \rightarrow \infty$. In the hierarchical construction presented in Sec. IV, $K$ is finite and the Matsubara terms $m$ $=1, \ldots, M$ are also finite. The residue correction due to the small difference between the exact $C_{a}(t)$ and the parametrized ones with finite terms is considered in Sec. V. By doing that, we can even treat the dissipation at $T=0$, where the FDT of Eq. (A1) assumes

$$
C_{\boldsymbol{a}}(t)=\frac{1}{\pi} \int_{0}^{\infty} d \omega e^{-i \omega t} J_{\boldsymbol{a}}(\omega), \quad \text { for } T=0,
$$

while the parametrized ones used in Sec. IV are at a certain finite low temperature.

\section{APPENDIX B: RECURSIVE GREEN'S FUNCTIONS, MEMORY KERNELS, AND CONTINUED FRACTION FORMALISM}

\section{EOM in tridiagonal coupling matrix form}

For the case of a single-mode Drude-Debye dissipation at high temperature, the EOM assumes the standard tridiagonal coupling matrix form-i.e., Eqs. (31) in the special case of a single mode. This property has been used to construct the continued-fraction formalism [19-22]. The Green's functions involved are also identified, together with an interesting application to the establishment of an analytical expression for electron transfer rate processes [36].

Here, we would like to extend our previous continuedfraction Green's-function formalism to the present complex dissipation systems. To that end, we shall first recast Eqs.
(45) in the standard tridiagonal coupling matrix form. This is done by considering the order of $\rho_{\mathrm{n}}$ depending on the hierarchy generating functionals:

$$
n_{\mathrm{n}} \equiv \sum_{a, k}\left(n_{2 k}^{a}+n_{2 k+1}^{a}+\bar{n}_{2 k}^{a}+\bar{n}_{2 k+1}^{a}\right)+\sum_{a} n_{\mathrm{D}}^{a}+\sum_{a, m} \check{n}_{m}^{a} .
$$

Collect now all those $n$ th-tier auxiliary density operatorsi.e., $\rho_{\mathrm{n}}$, which have $n_{\mathrm{n}}=n$ - and arrange them into a vector $\boldsymbol{\rho}_{n} \equiv\left\{\rho_{\mathrm{n}} ; n_{\mathrm{n}}=n\right\}$. The size of this vector is $\frac{(n+P-1) !}{n !(P-1) !}$ if there are no truncations involved; see Sec. V B. Here, $P$ is the number of non-negative integers in the index set $\mathrm{n}$; see the comments after Eq. (44).

The hierarchical EOM [Eqs. (45)] can then be rearranged into the standard tridiagonal matrix form as

$$
\partial_{t} \boldsymbol{\rho}_{n}=-\boldsymbol{\Lambda}_{n} \boldsymbol{\rho}_{n}-i \boldsymbol{A}_{n} \boldsymbol{\rho}_{n-1}-i \boldsymbol{B}_{n} \boldsymbol{\rho}_{n+1} .
$$

Note that the swap ones $\rho_{\mathrm{n}}^{\{\leftrightharpoons\}}$ are now a part of $\boldsymbol{\rho}_{n}$ and the $\boldsymbol{\Lambda}_{n}$ matrix elements are all numbers, except the Liouvillian $\mathcal{L}$, which can also be time dependent in the presence of external pulsed fields, for example. All $\rho_{\mathrm{n}}^{\{-\}}$are arranged into $\boldsymbol{\rho}_{n-1}$ with the Liouville-space operators defined in Eqs. (50) involved in the elements of $\boldsymbol{A}_{n}$. Those $\rho_{\mathrm{n}}^{\{+\}}$are now in $\boldsymbol{\rho}_{n+1}$ and the elements in $\boldsymbol{B}_{n}$ are according to Eq. (49).

Note that $\rho_{0}(t)=\rho(t)$ is the reduced density operator of primary interest and $\boldsymbol{\Lambda}_{0}=i \mathcal{L}$. The collection of the $n$ th-tier auxiliary density operators has the leading contributions of the $(2 n)$ th-order in the overall system-bath couplings to the $\rho(t)$ of primary interest. The initial conditions of Eqs. (B1) are $\boldsymbol{\rho}_{n}\left(t_{0}\right)=\delta_{n 0} \rho\left(t_{0}\right)$, as inferred from the definition.

\section{Green's functions versus memory kernels}

In terms of the propagators, by which

$$
\boldsymbol{\rho}_{n}(t) \equiv \mathcal{U}_{n}\left(t, t_{0}\right) \rho\left(t_{0}\right),
$$

Eqs. (B1) read [noting that $\mathcal{U}_{n}\left(t_{0}, t_{0}\right) \equiv \delta_{n 0}$ ]

$$
\partial_{t} \mathcal{U}_{n}=-\boldsymbol{\Lambda}_{n} \mathcal{U}_{n}-i \boldsymbol{A}_{n} \mathcal{U}_{n-1}-i \boldsymbol{B}_{n} \mathcal{U}_{n+1}
$$

Introduce now the hierarchical set of Green's functions in the reduced system Liouville space as follows:

$$
\begin{gathered}
\mathcal{U}_{0}\left(t, t_{0}\right) \equiv \mathcal{G}_{0}\left(t, t_{0}\right) \\
\mathcal{U}_{n}\left(t, t_{0}\right) \equiv-i \int_{t_{0}}^{t} d \tau \mathcal{G}_{n}(t, \tau) \boldsymbol{A}_{n} \mathcal{U}_{n-1}\left(\tau, t_{0}\right), \quad n \geqslant 1 .
\end{gathered}
$$

The initial conditions are $\mathcal{G}_{n}\left(t_{0}, t_{0}\right)=1$. Substituting Eqs. (B4) into Eq. (B3) then leads to

$$
\partial_{t} \mathcal{G}_{n}\left(t, t_{0}\right)=-\boldsymbol{\Lambda}_{n} \mathcal{G}_{n}\left(t, t_{0}\right)-\int_{t_{0}}^{t} d \tau \boldsymbol{\Pi}_{n}(t, \tau) \mathcal{G}_{n}\left(\tau, t_{0}\right),
$$

with the involving memory kernel of

$$
\Pi_{n}(t, \tau)=\boldsymbol{B}_{n} \mathcal{G}_{n+1}(t, \tau) \boldsymbol{A}_{n+1} .
$$


In particular, $\Pi_{0}(t, \tau) \equiv \Pi(t, \tau)$ is the primary memory kernel, by which (noting that $\Lambda_{0}=i \mathcal{L}$ )

$$
\dot{\rho}(t)=-i \mathcal{L}(t) \rho(t)-\int_{t_{0}}^{t} d \tau \Pi(t, \tau) \rho(\tau) .
$$

For the time-independent system Hamiltonian, $\mathcal{G}_{n}(t, \tau)$ $=\mathcal{G}_{n}(t-\tau)$ and $\boldsymbol{\Pi}_{n}(t, \tau)=\boldsymbol{\Pi}_{n}(t-\tau)$. The above Green's functions (or memory kernels) can be resolved in the Laplace frequency domain via the continued-fraction expression of $[36,37]$

$$
\hat{\mathcal{G}}_{n}(s)=\frac{1}{s+\boldsymbol{\Lambda}_{n}+\boldsymbol{B}_{n} \hat{\mathcal{G}}_{n+1}(s) \boldsymbol{A}_{n+1}} .
$$

Unlike the auxiliary propagators $\mathcal{U}_{n}$, which couple with both $\mathcal{U}_{n+1}$ and $\mathcal{U}_{n-1}$, the Green's functions $\mathcal{G}_{n}$ couple only with $\mathcal{G}_{n+1}$. Thus, the evaluation of the reduced dynamics of primary interest could be more efficiently carried out in terms of the auxiliary Green's functions, especially when there is no time-dependent external fields. The continued-fraction formalism, combined with the Dyson-equation technique, has been recently applied to the simple electron transfer, a spin-boson system, in Drude-Debye solvents [36,37].
[1] R. P. Feynman and F. L. Vernon, Jr., Ann. Phys. (N.Y.) 24, 118 (1963).

[2] A. G. Redfield, Adv. Magn. Reson. 1, 1 (1965).

[3] R. Kubo, M. Toda, and N. Hashitsume, Statistical Physics II: Nonequilibrium Statistical Mechanics, 2nd ed. (SpringerVerlag, Berlin, 1985).

[4] U. Weiss, Quantum Dissipative Systems, 2nd ed., Series in Modern Condensed Matter Physics, Vol. 10 (World Scientific, Singapore, 1999).

[5] H. Kleinert, Path Integrals in Quantum Mechanics, Statistics, Polymer Physics, and Financial Markets, 4th ed. (World Scientific, Singapore, 2006).

[6] M. B. Plenio and P. L. Knight, Rev. Mod. Phys. 70, 101 (1998).

[7] T. Dittrich, P. Hänggi, G. L. Ingold, B. Kramer, G. Schön, and W. Zwerger, Quantum Transport and Dissipation (WileyVCH, Weinheim, 1998).

[8] M. Thorwart, M. Grifoni, and P. Hänggi, Ann. Phys. (N.Y.) 293, 15 (2001).

[9] H. P. Breuer and F. Petruccione, The Theory of Open Quantum Systems (Oxford University Press, New York, 2002).

[10] H. Grabert, P. Schramm, and G. L. Ingold, Phys. Rep. 168, 115 (1988).

[11] A. O. Caldeira and A. J. Leggett, Ann. Phys. (N.Y.) 149, 374 (1983); 153, 445 (1984).

[12] A. J. Leggett, S. Chakravarty, A. T. Dorsey, and M. Gary, Rev. Mod. Phys. 59, 1 (1987); 67, 725(E) (1995).

[13] Y. J. Yan, Phys. Rev. A 58, 2721 (1998).

[14] Y. J. Yan, F. Shuang, R. X. Xu, J. X. Cheng, X. Q. Li, C. Yang, and H. Y. Zhang, J. Chem. Phys. 113, 2068 (2000).

[15] R. X. Xu and Y. J. Yan, J. Chem. Phys. 116, 9196 (2002).

[16] Y. J. Yan and R. X. Xu, Annu. Rev. Phys. Chem. 56, 187 (2005).
[17] R. X. Xu, P. Cui, X. Q. Li, Y. Mo, and Y. J. Yan, J. Chem. Phys. 122, 041103 (2005).

[18] N. Makri, J. Math. Phys. 36, 2430 (1995).

[19] Y. Tanimura and R. Kubo, J. Phys. Soc. Jpn. 58, 101 (1989).

[20] Y. Tanimura and P. G. Wolynes, Phys. Rev. A 43, 4131 (1991).

[21] A. Ishizaki and Y. Tanimura, J. Phys. Soc. Jpn. 74, 3131 (2005).

[22] Y. Tanimura, J. Phys. Soc. Jpn. 75, 082001 (2006).

[23] J. S. Shao, J. Chem. Phys. 120, 5053 (2004).

[24] Y. A. Yan, F. Yang, Y. Liu, and J. S. Shao, Chem. Phys. Lett. 395, 216 (2004).

[25] J. S. Shao, Chem. Phys. 322, 187 (2006).

[26] J. T. Stockburger and C. H. Mak, J. Chem. Phys. 110, 4983 (1999).

[27] J. T. Stockburger and H. Grabert, Phys. Rev. Lett. 88, 170407 (2002).

[28] W. T. Strunz and T. Yu, Phys. Rev. A 69, 052115 (2004).

[29] T. Yu, Phys. Rev. A 69, 062107 (2004).

[30] P. Hänggi and G. L. Ingold, Chaos 15, 026105 (2005).

[31] B. L. Hu, J. P. Paz, and Y. Zhang, Phys. Rev. D 45, 2843 (1992).

[32] R. Karrlein and H. Grabert, Phys. Rev. E 55, 153 (1997).

[33] J. S. Jin, S. Welack, J. Y. Luo, X. Q. Li, P. Cui, R. X. Xu, and Y. J. Yan, J. Chem. Phys. 126(12) (to be published).

[34] A. Thielmann, M. H. Hettler, J. König, and G. Schön, Phys. Rev. Lett. 95, 146806 (2005).

[35] P. Cui, X. Q. Li, J. S. Shao, and Y. J. Yan, Phys. Lett. A 357, 449 (2006).

[36] P. Han, R. X. Xu, B. Q. Li, J. Xu, P. Cui, Y. Mo, and Y. J. Yan, J. Phys. Chem. B 110, 11438 (2006).

[37] P. Han, R. X. Xu, P. Cui, Y. Mo, G. Z. He, and Y. J. Yan, J. Theor. Comput. Chem. 5, 685 (2006).

[38] C. Meier and D. J. Tannor, J. Chem. Phys. 111, 3365 (1999). 